\title{
Cabazitaxel for metastatic castration-resistant prostate cancer: safety data from the Spanish expanded access program
}

\author{
Daniel Castellano ${ }^{\dagger}$, Luis M Antón Aparicio, Emilio Esteban, Alfredo Sánchez- \\ Hernández, José Ramón Germá, Norberto Batista, Pablo Maroto, Begoña Pérez- \\ Valderrama, Raquel Luque, María José Méndez-Vidal \& on behalf of the cabazitaxel \\ EAP study
}

Hniversity Hospital 12 de Octubre, Medical Oncology Department, Urooncology Unit (I+12 Research Institute), Madrid, Spain

\begin{abstract}
Background: Based on the TROPIC study results, cabazitaxel was approved for the management of metastatic castration-resistant prostate cancer (mCRPC) progressing on or after docetaxel.

Methods: This multi-centre program provided early access to cabazitaxel to patients with mCRPC before its commercialization. Safety data from 153 Spanish patients receiving cabazitaxel $25 \mathrm{mg} / \mathrm{m}^{2}$ i.v. Q3W, plus oral prednisone/prednisolone $10 \mathrm{mg}$ daily, are reported.

Results: Median age of patients was 70 years (26.8\% $\geq 75$ years), 94.1 and $26.8 \%$ had bone and visceral metastasis, respectively. Most had an Eastern Cooperative Oncology Group $\leq 1(88.9 \%)$ and had received a median of 8.0 cycles of last docetaxel treatment. The median of cabazitaxel cycles and cumulative dose were 6.0 (Interquartile range [IQR]: $4.0 ; 8.0$ ) and 148.9 (IQR: $98.2 ; 201.4) \mathrm{mg} / \mathrm{m}^{2}$, respectively. Adverse events (AEs) possibly related to cabazitaxel occurred in $143(93.5 \%)$ patients. The most frequent grade $\geq 3$ AEs were neutropenia $(\mathrm{n}=25,16.3 \%)$ and asthenia $(\mathrm{n}=17,11.1 \%)$. Febrile neutropenia and grade $\geq 3$ diarrhea occurred in $5.2 \%$ of the patients each. There were five $(3.3 \%)$ possibly treatment-related deaths, mainly infection-related. G-CSFs were used in $114(74.5 \%)$ patients, generally as prophylaxis $(\mathrm{n}=107 ; 69.9 \%)$. Grade $\geq 3$ peripheral neuropathy and nail disorders were uncommon.

Conclusions: Cabazitaxel administration, in a real-world setting, is tolerated by Spanish patients with mCRPC, and the AEs are manageable.
\end{abstract}

Keywords: cabazitaxel, compassionate use studies, docetaxel, prostatic neoplasms, safety 


\section{Introduction}

Docetaxel-based chemotherapy is the standard first-line therapy for metastatic castration-resistant prostate cancer (mCRPC); however, it is not curative and after initial response many patients progress. Until recently, second-line chemotherapy for mCRPC patients was an unmet clinical need for a rapidly progressing and debilitating disease, although results from the TROPIC study with cabazitaxel, a next generation taxane designed to overcome drug resistance to docetaxel, prompted its approval as secondline chemotherapy for mCRPC.

Cabazitaxel is a tubulin-binding taxane that promotes the microtubules stabilization leading to mitotic block and apoptosis of tumor cells [1], but also interferes with androgen receptor (AR)-signaling pathway, thus preventing AR nuclear translocation as suggested in recent studies [2,3]. Cabazitaxel demonstrated to provide a survival benefit over mitoxantrone in the randomized, Phase III TROPIC study in $755 \mathrm{mCRPC}$ patients progressing from a docetaxel-containing regimen [4]. The median overall survival was in favor of cabazitaxel arm (15.1 months) versus the mitoxantrone arm (12.7 months) (Hazard ratio [HR]: $0.70,95 \%$ CI $0.59-0.83, \mathrm{p}<0.0001$ ), resulting in a $30 \%$ reduction in the risk of death. The PFS was also in favor of cabazitaxel arm with 2.8 versus 1.4 months (HR: $0.74,95 \%$ CI $0.64-$ $0.86, \mathrm{p}<0.0001)$. Nevertheless, in the TROPIC study significant hematological adverse events (AEs) (neutropenia, febrile neutropenia and neutropenic complications) were commonly reported in the cabazitaxel group, often during cycle 1. Also nonhematological AEs such as diarrhea, fatigue, asthenia and back pain were frequent. Although toxicities typical of the taxane class (neutropenia and its consequences, diarrhea) are usually predictable and manageable, a need to raise awareness of those risks and their management amongst clinicians was stated. The proactive management of AEs relative to cabazitaxel by the appropriate secondary prophylaxis with G--CSF, as per American Society of Clinical Oncology (ASCO) guidelines and EORTC guidelines, should be promoted as well to reduce the risk of neutropenic complications $[5,6]$.

Because of the survival benefit observed from the TROPIC study, there was a significant interest and augmented demand for access to cabazitaxel until it was commercially available. Therefore, this international, single-arm, multi-centre, open-label study (NCT01254279) provided early access to cabazitaxel for use in patients with baseline and clinical characteristics similar to the patients evaluated in the TROPIC study, with the objective of further evaluate the cabazitaxel safety profile in the real-life setting and across different geographical areas. The results from an interim analysis, performed on December 2012, of the expanded access program (EAP) in Spain are described in this article.

\section{Patients and methods}

\subsection{Eligibility criteria}

Patients with mCRPC who had progressed during or after treatment with a docetaxel-containing regimen for $\mathrm{mCRPC}$ were enrolled at selected sites in Spain. Other eligibility criteria included: age $\geq 18$ years; an Eastern Cooperative Oncology Group (ECOG) performance status of $\leq 2$; previous or ongoing surgical (orchiectomy) or medical castration; life expectancy of $\geq 3$ months; and adequate bone marrow, liver and renal function. Exclusion criteria were the same as those of the TROPIC study [4].

The study was performed after approval by an Independent Ethics Committee of each site and in accordance with the Declaration of Helsinki, Good Clinical Practices, and local ethical and legal requirements. Signed informed consent was obtained from all patients before study entry. 


\subsection{Treatment plan and study assessments}

Eligible patients received cabazitaxel $25 \mathrm{mg} / \mathrm{m}^{2}$ intravenously over $1 \mathrm{~h}$ every 3 weeks, in combination with oral prednisone or prednisolone $10 \mathrm{mg}$ daily until disease progression, death, unacceptable toxicity or because of the investigator's decision. Patient recruitment was stopped once cabazitaxel was commercially available.

Safety assessments (hematology and biochemistry) were performed before each cycle. Patients were evaluated for AEs during therapy and until 30 days after the last study drug dose. AEs were graded using the National Cancer Institute common toxicity criteria (NCI-CTCAE) version 4.0 [7], and summarized using the Medical Dictionary for Regulatory Activities (MedDRA) version 15.0 terminology [8].

When necessary, a treatment delay due to toxicity of up to 2 weeks or one dose reduction (to 20 $\mathrm{mg} / \mathrm{m}^{2}$ for cabazitaxel) per patient was permitted. Proactive management of AEs relative to cabazitaxel, primarily neutropenia and related disorders, was recommended in line with the ASCO guidelines [5]. Primary prophylaxis with G-CSF was considered in patients with high-risk features (including but not limited to age > 65 years, poor-performance status or prior febrile neutropenia episodes) according to ASCO guidelines starting from first cycle of treatment. Secondary prophylaxis with G-CSF was also recommended in case of neutropenic complication in earlier cycle of chemotherapy without primary prophylaxis.

\subsection{Statistical considerations}

The primary end point was to document the overall safety of cabazitaxel in mCRPC patients who had progressed during or after treatment with a docetaxel-containing regimen. A descriptive safety analysis, including number of cycles, cumulative dose received, reason for end of treatment, AEs and serious adverse events, was performed on the safety population (all enrolled patients receiving at least part of one dose of cabazitaxel).

Additionally, the cabazitaxel efficacy (prostate-specific antigen [PSA] response and biochemical progression free survival) was analyzed in a cohort of patients participating in the Spanish arm of the EAP. For this efficacy subanalysis, the patients were selected from the sites with higher recruitment rate and no formal selection was used.

\section{Results}

\subsection{Patients and eligibility}

From March 2011 to August 2011, 153 patients enrolled into the study at 25 sites in Spain and whose first treatment was taken before or on May 302012 were included in the interim analysis. Their characteristics are summarized in Table 1. Median age was 70 (interquartile range [IQR]: 65 - 75) years; $26.8 \%$ were aged 75 years or more; $94.1 \%(n=144)$ had bone metastasis and $26.8 \%$ had visceral $(n=41)$ metastasis. 
Table 1. Patient baseline characteristics.

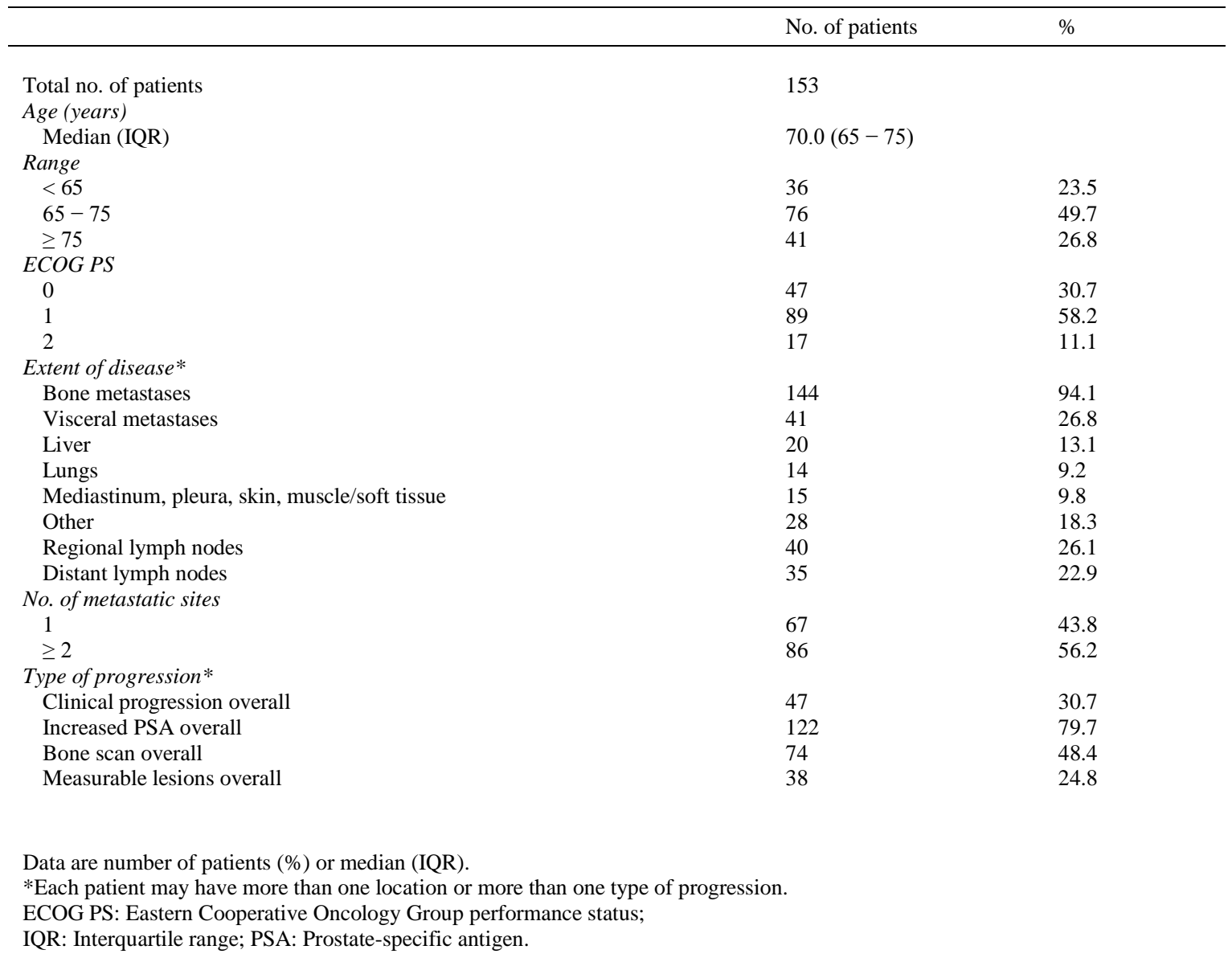

Patients had previously received a median of 8.0 (IQR: 6.0; 10.0) cycles of last docetaxel. Docetaxel was received as firstline treatment in 72 patients. Disease progression occurred at a median time of 6.5 months (IQR: 2.5; 12.1) from last docetaxel dose with 59.5\% ( $\mathrm{n}=91)$ patients experiencing disease progression $\geq 3$ months from last docetaxel dose. The proportion of patients receiving first cabazitaxel dose within or after 6 months of last docetaxel dose was similar (Table 2). 
Table 2. Previous treatment with docetaxel-containing regimen.

\begin{tabular}{|c|c|c|}
\hline & No. of patients & $\%$ \\
\hline Total no. of patients & 153 & \\
\hline Median (IQR) & $1.0(1.0-2.0)$ & \\
\hline \multicolumn{3}{|l|}{ No. cycles of last docetaxel administration } \\
\hline Median (IQR) & $8.0(6.0-10.0)$ & \\
\hline \multicolumn{3}{|c|}{ Distribution of docetaxel cumulative dose $\left(\mathrm{mg} / \mathrm{m}^{2}\right) *$} \\
\hline$<225$ & 9 & 6.0 \\
\hline $225-450$ & 29 & 19.5 \\
\hline $451-675$ & 69 & 46.3 \\
\hline $676-900$ & 24 & 16.1 \\
\hline$\geq 3$ months from last dose & 91 & 59.5 \\
\hline \multicolumn{3}{|c|}{ Time from last docetaxel dose to disease progression $(\text { months })^{\S}$} \\
\hline Median (IQR) & $6.5(2.5 ; 12.1)$ & \\
\hline \multicolumn{3}{|c|}{ Time elapsed from last docetaxel dose to first cabazitaxel dose } \\
\hline within 6 months since last docetaxel dose & 72 & 47.4 \\
\hline$>6$ months since last docetaxel dose & 80 & 52.6 \\
\hline
\end{tabular}

* 4 missing.

$\$ 1$ missing.

$\S$ For pats who progressed after last docetaxel dose.

IQR: Interquartile range.

\subsection{Treatment exposure}

Patients received a median number of 6.0 (IQR: 4.0; 8.0) cycles of cabazitaxel and a median cumulative dose of 148.9 (IQR: $98.2 ; 201.4) \mathrm{mg} / \mathrm{m}^{2}$ (Table 3). A hundred of patients (65.4\%) completed 6 cycles and $27(17.6 \%)$ patients received up to 10 cycles of treatment with one patient receiving 17 cycles.

At the cut-off date of the data interim analysis (May, 2012), 21 patients were still under treatment in the EAP in Spain, and cabazitaxel treatment was ended in 132 patients. Cabazitaxel dose reduction was necessary in 25 cycles in 24 patients of the 132 patients who ended treatment (18.2\%) due to nonhematological (7.6\% patients), hematological (4.5\% patients) or both (3.8\% patients) cabazitaxelrelated AEs. A total of 77 cycles were delayed in 54 patients (40.9\%), due to non-hematological AEs (11.4\% patients), hematological AEs (6.1\% patients) or both toxicities (2.3\% patients) and in 29 patients $(22 \%)$ for non drug-related reasons (i.e., administrative issues, holidays, patient's request or investigator's decision). The median relative dose intensity was equivalent to $99.7 \%$ (IQR: 97.9; 100.3) of the predicted dose intensity.

Main reasons for discontinuation were disease progression $(n=64 ; 48.5 \%)$, AEs $(n=36 ; 27.3 \%)$, investigator's decision $(\mathrm{n}=24,18.2 \%)$, patient's decision $(\mathrm{n}=5 ; 3.8 \%)$, treatment completed $(\mathrm{n}=1$; $0.8 \%)$, bad tolerance $(n=1 ; 0.8 \%)$ or lost to follow-up $(n=1 ; 0.8 \%)$. The Investigator decided to discontinue treatment in $15(11.4 \%)$ patients because the best clinical benefit was achieved or because they have completed 10 cycles of cabazitaxel treatment. 
Table 3. Cabazitaxel treatment received.

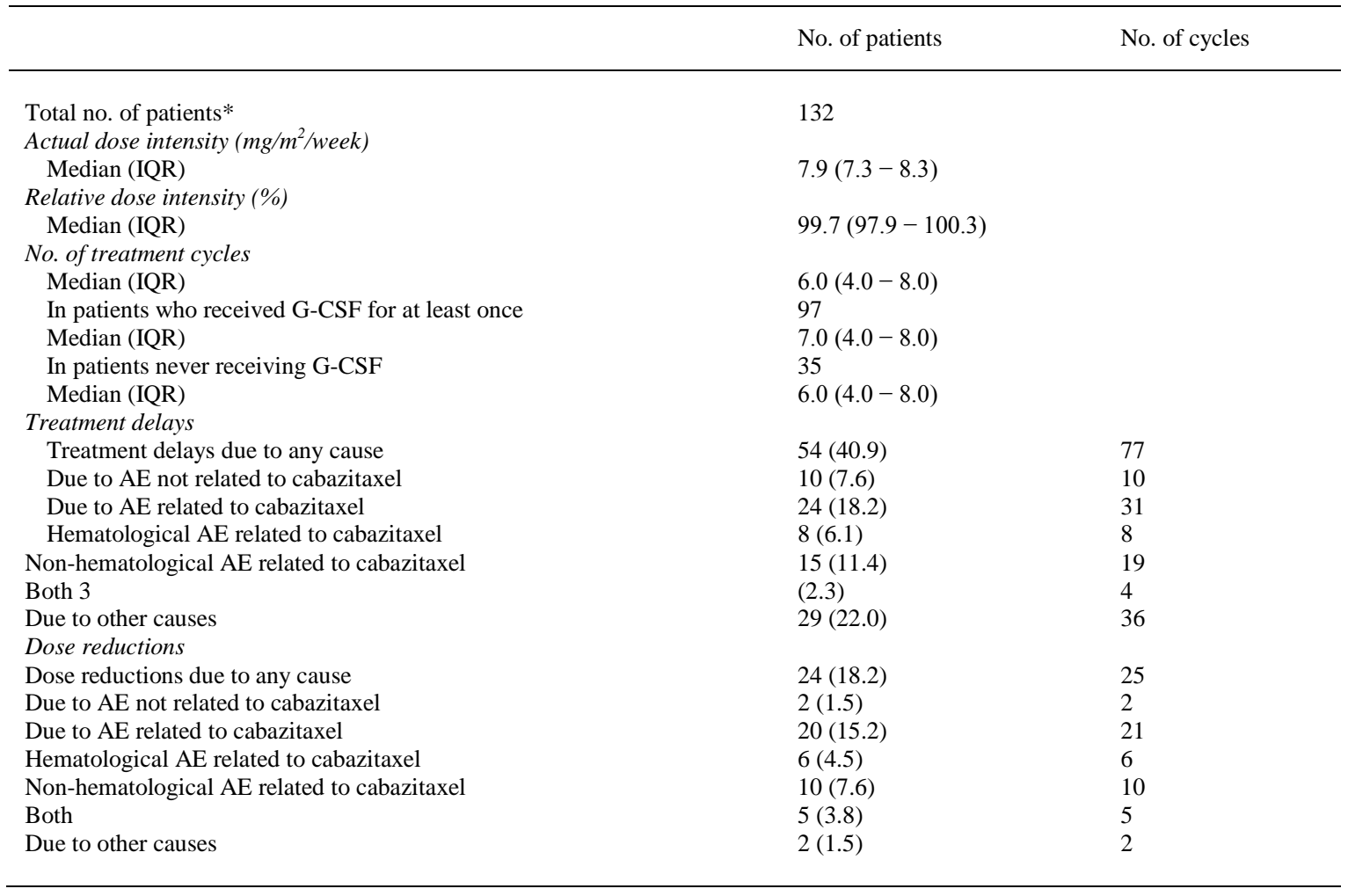

Data are number of cycles or median (IQR)

*Patients who ended cabazitaxel treatment at the interim analysis cut-off date.

AE: Adverse event; IQR: Interquartile range.

\subsection{Safety}

All 153 patients were included in the safety analysis as they received at least one dose of treatment. One hundred and forty three patients $(93.5 \%)$ reported possibly treatment related AEs. Possible related AEs observed in $>20 \%$ of patients were mainly general disorders (asthenia [62.7\%]), gastrointestinal (GI) (diarrhea [45.8\%], nausea [22.2\%] and decreased appetite [22.2\%]) or hematological (anemia [37.9\%] and neutropenia [22.2\%]).

Grade $\geq 3$ AEs were recorded in 66 patients $(43.1 \%)$. The most common clinically significant grade $\geq$ 3 AEs were neutropenia (25 [16.3\%] patients) and asthenia (17 [11.1\%] patients) (Table 4). Grade $\geq 3$ febrile neutropenia and diarrhea occurred in $8(5.2 \%)$ patients each. Although direct comparisons are not feasible, the percentage of patients reporting hematological grade $3-4$ AEs differ from those in the TROPIC study (Figure 1) possibly due to prophylactic use of G-CSF. Grade $\geq 3$ peripheral neuropathy ( 1 $[0.7 \%])$ were uncommon. No grade $\geq 3$ nail disorders were reported. 
Table 4. Patients with at least one treatment-related TEAE.

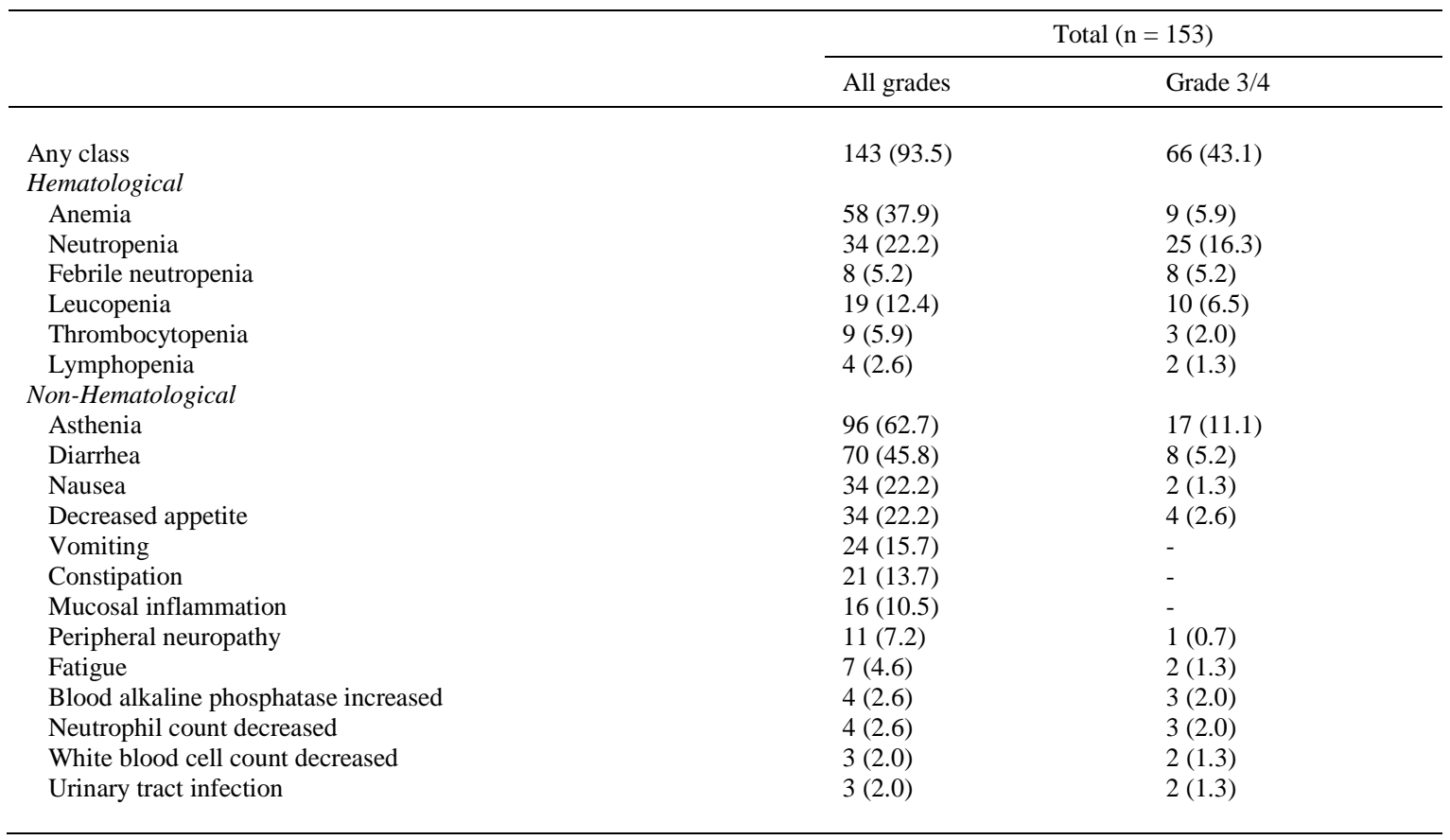

Data are number of patients (\%). TEAEs were graded according to the National Cancer Institute Common Terminology Criteria for Adverse Events (version 4.0)

and summarized with the Medical Dictionary for Regulatory Activities terminology (version 15.0). Events listed are those occurring at grade 3 or higher severity

in $\geq 1 \%$ of patients and/or at all grades in $\geq 10 \%$ of patients.

TEAES: Treatment emergent adverse events.

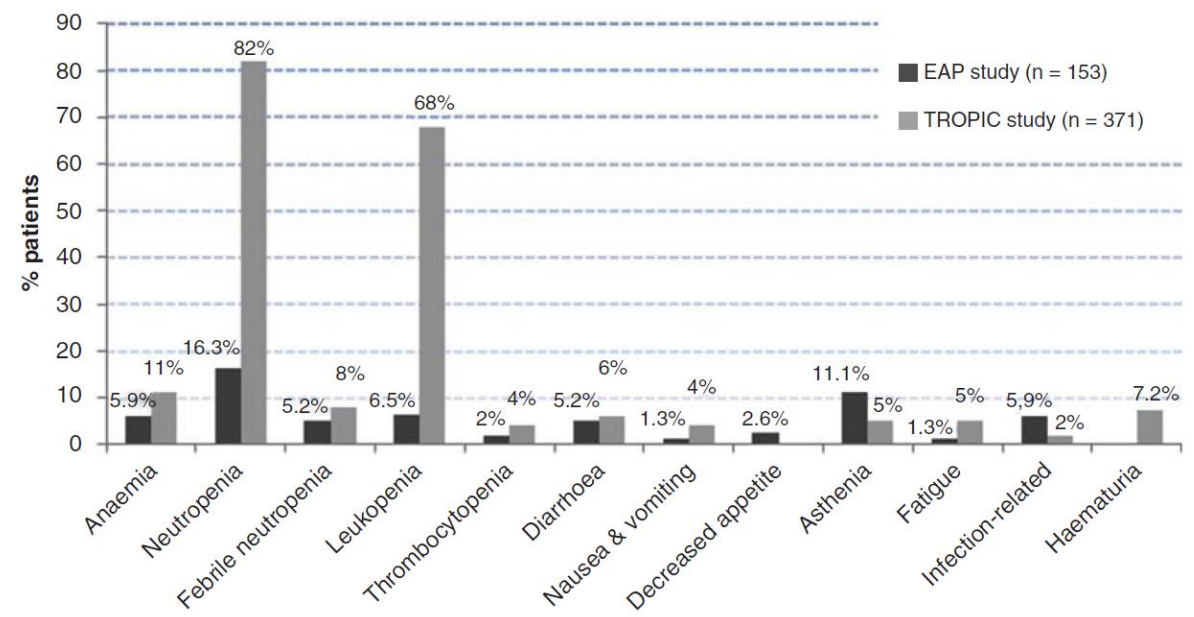

Figure 1. Grade $\$ 3$ treatment emergent adverse events (TEAEs) reported in $\$ 2 \%$ of patients in the Spanish EAP and in the TROPIC study.

EAP: Expanded access program. 
Cabazitaxel treatment was discontinued in 36 (27.3\%) patients due to AEs. Most common AEs leading to discontinuation were grade $3 / 4$ infection-related disorders $(n=9 ; 5.9 \%)$, grade $3 / 4$ general disorders and administration site conditions $(n=5 ; 3.3 \%)$ and grade $3 / 4$ GI disorders mainly diarrhea $(n=$ $4,2.6 \%)$.

There were five $(3.3 \%)$ possibly treatment-related deaths, mainly infection-related: urinary tract infection $(n=1)$, neutropenic infection $(n=1)$, peritonitis $(n=1)$, septic shock $(n=1)$ and febrile neutropenia $(n=1)$. Four out of the five patients were aged $\geq 70$ years. All had received $\leq 2$ cycles of treatment (drug exposure: 1 day $[\mathrm{n}=2] ; 22$ days $[\mathrm{n}=2]$ and 30 days $[\mathrm{n}=1]$ ).

G-CSF were used in $114(74.5 \%)$ patients mostly as preventive treatment $(n=107 ; 69.9 \%)$, with 101 patients receiving G-CSF at cycle 1 . Of 101 patients, 88 received G-CSF as primary prophylaxis at first cycle (Table 5).

Table 5. Prevention of neutropenia with G-CSF in high-risk patients.

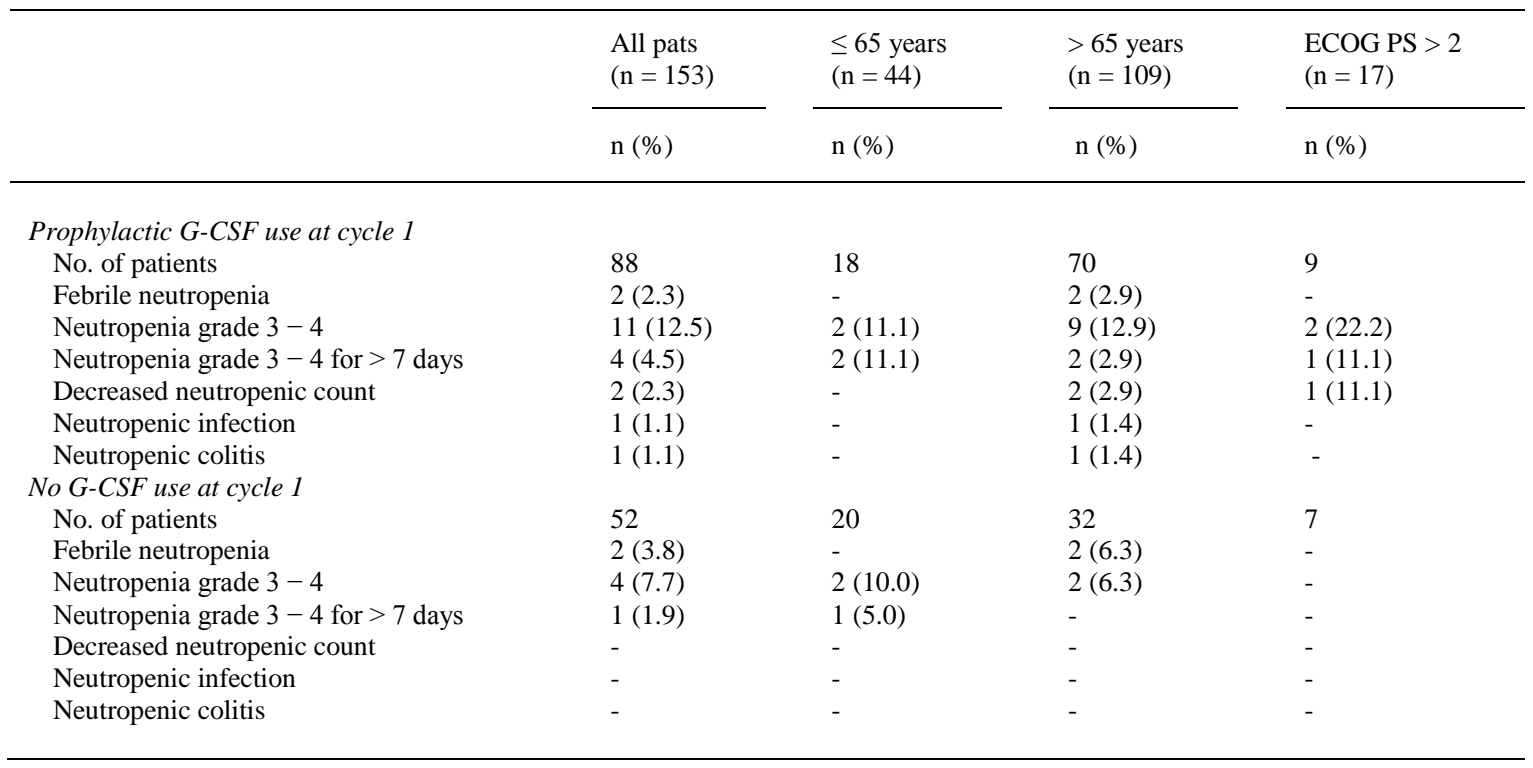

ECOG PS: Eastern Cooperative Oncology Group performance status.

\subsection{Efficacy subanalysis: PSA response}

Additionally, the PSA response and biochemical progression free survival after cabazitaxel treatment was analyzed outside the EAP protocol in a cohort of 65 patients from 6 sites participating in the Spanish arm of the EAP. Median baseline PSA was $864 \mathrm{ng} / \mathrm{ml}$. A PSA response to cabazitaxel (PSA reduction of $>50 \%$ ) was achieved in 47.7\% (31 out of 65) patients. Median progression-free survival was 4.4 months (range: $2.7-6.1$ ) [9]. 


\section{Discussion}

The results from our study further evaluate the cabazitaxel safety profile in mCRPC patients from Spain participating in the EAP program. These results are particularly relevant as they provide safety and efficacy data in a real-life setting, which does not differ from that observed in the TROPIC study.

Data from the Spanish EAP suggest a good safety profile of cabazitaxel, with asthenia, myelosuppression (i.e., anemia, neutropenia) and GI symptoms (diarrhea, nausea and decreased appetite) as common toxicities (> $20 \%$ of patients), which is consistent with the results reported in the TROPIC study. Besides, cabazitaxel treatment was well tolerated, with $<30 \%$ of patients discontinuing treatment due to AEs. A majority of patients completed 6 cycles of cabazitaxel treatment and almost $20 \%$ received up to 10 cycles of treatment. In addition, most patients received the full dose of study treatment (median relative dose intensity was almost $100 \%$ of the predicted dose intensity).

The exposure to treatment and the sources for AE collection in the Spanish EAP (single-arm, compassionate-use) and the TROPIC (randomized, Phase III) studies are not comparable, thus a direct comparison is not possible. However, despite having a patient population that mirrored that of the TROPIC study, cabazitaxel seems to be better tolerated in our study than in the TROPIC study possibly due to preventive toxicity management. The percentage of patients reporting hematological grade $\geq 3$ AEs, namely grade $\geq 3$ neutropenia and febrile neutropenia, was considerably reduced in the EAP in Spain (16.3 and 5.2\% patients, respectively) compared to the TROPIC study ( 82 and $8 \%$ patients, respectively). A more frequent follow-up for neutropenia was made in the TROPIC (weekly) than in the EAP (every 3 weeks) study, which might partly explain the difference in the number of neutropenia cases. The percentage of infection-related AEs was slightly higher in our study (5.9 vs $2 \%$ in the TROPIC study), but even if we include these infection-related AEs within the group of neutropenic complications the hematological safety profile of cabazitaxel continues to be better in the EAP in Spain (27.4 vs $92 \%$ in the TROPIC study). In addition, no cases of grade $\geq 3$ peripheral neuropathy or arthralgia/back pain were reported here and the rate of grade $\geq 3$ diarrhea was slightly lower than that reported in the TROPIC study. Grade $\geq 3$ asthenia/fatigue (12.4\%), which is commonly reported with cytotoxic chemotherapy [10], did not differ from that of the TROPIC study [4].

The key safety data for cabazitaxel in mCRPC were derived from the TROPIC study; however, the results observed in our real-world Spanish population demonstrate that cabazitaxel toxicities are usually predictable and manageable in clinical practice. The main characteristics of the study population in the EAP study compared to the TROPIC study, with a higher proportion of patients aged $>75$ years (26.8 vs $18 \%$, respectively) and with more advanced disease (94 vs $80 \%$ with bone metastasis, respectively), cannot explain the differences in the safety profiles. The proactive management of certain clinical factors, which predispose to increased complications from prolonged neutropenia, such as age $>65$ years or poor performance status $(E C O G \geq 2$ ), probably have played a role for minimization of hematological AEs. Investigators participating in our study had extensive experience in administering taxanes and were instructed in the use of appropriate prophylaxis with G-CSF to reduce the risk of neutropenic complications from the first cycle of treatment [5]. In line with this, Di Lorenzo et al. suggested a marked decrease in the relative risk of grade $\geq 3$ neutropenia/febrile neutropenia per cycle per patient (by approximately seven times) with cabazitaxel if prophylaxis with PEG-filgrastim was used [11]. Investigators were also required to strictly follow the information provided by the Sponsor for cabazitaxel dose reduction, interruption or delay. Educational local programs might be promoted in clinical practice, which include the use of the appropriate cabazitaxel dose modifications recommended by the Sponsor as required, the use of preventive strategies to avoid cabazitaxel AEs, and careful patient education on symptom recognition, self-care and clear instructions for seeking advice for an effective management of AEs [12].

The safety results from the EAP in other countries indicate that cabazitaxel has an acceptable tolerability in the routine clinical practice setting across the globe [11,13-19]. Preliminary safety results of the European EAP program has shown that prophylactic use of G-CSF, especially at cycle 1 improves tolerability in patients aged $\geq 75$ years treated with cabazitaxel. In our study, the number of patients aged 
$\geq 75$ years $(n=41)$ was too low to be analyzed. In addition, data on the impact of cabazitaxel treatment in patient's quality of life has been collected within the EAP in several countries like UK or Canada, and preliminary results are promising [13,17]. Final results from the whole international EAP program are awaited with interest.

On the other hand, our study population was representative of the 'unselected' population of mCRPC patients attended in daily clinical practice which usually have poor prognostic factors: aged > 75 years; presenting bone or visceral metastasis (including liver metastasis in 13\%); with two or more metastatic sites involved; with bone-scan progression or measurable disease progression [20,21]. Pre-treatment with docetaxel, that is, cumulative dose and median number of cycles of, received by the patients in our study was equivalent to that of the TROPIC study. Bearing in mind that this study was not designed to evaluate efficacy, the PSA response of cabazitaxel in a cohort of 65 patients demonstrated a similar efficacy of cabazitaxel to that observed in the TROPIC study (median progression-free survival 4.4 vs 2.8 months, respectively). Actually, in $11.4 \%$ of patients the treatment was discontinued because the best clinical benefit was achieved or because they have completed 10 cycles of cabazitaxel treatment. A recent analysis by Bahl et al. of the survival rates in the TROPIC study showed longer survival rates $(\geq 2$ vs $<2$ years) in patients receiving higher number of cabazitaxel cycles (median of 10 cycles vs median 6 cycles, respectively) [22].

\section{Conclusions}

In conclusion, the treatment of mCRPC with cabazitaxel is safe and tolerable with manageable AEs in the routine clinical practice, especially in a real-life Spanish population with poor prognostic factors (aged > 70; with visceral metastasis). Proactive management of AEs, especially in $>65$ years and ECOG $\geq 2$ patients is important and likely has a role for minimization of hematological AEs.

\section{Acknowledgements}

The physicians listed below cared for the patients in this study. The authors thank them for their cooperation and support: A. García Palomo. Hospital General de León, León; JA Arranz. Hospital General Universitario Gregorio Marañón, Madrid; M Doménech. Hospital Sant Joan de Deu, Althaia, Barcelona; JL González Larriba. Hospital Clínico San Carlos, Madrid; R López. Hospital Clínico Universitario Santiago, Santiago de Compostela; B. Mellado. Hospital Clínic Barcelona, Barcelona; A. Antón Torres. Hospital Universitario Miguel Servet, Zaragoza, Spain; R. Bastús. Mutua de Terrassa Barcelona, Spain; J. Cassinello. Hospital General de Guadalajara, Guadalajara; MA. Climent Duran. Instituto Valenciano de Oncología, Valencia; O. Juan. Hospital Arnau Vilanova, Valencia; M. López Brea. Hospital Marqués de Valdecilla, Santander; A Montesa. Hospital Carlos Haya Málaga; JL Pérez Gracia Clínica Universitaria de Navarra. Pamplona; F. Vázquez. H. Univ. Elche Alicante. The Study has been presented as Poster (P402.No. 2.895) at the European Cancer Conference 2013 (17th ECCO - 38 ${ }^{\text {th }}$ ESMO - 32nd ESTRO) held from 27 September - 1 October 2013 in Amsterdam, Netherlands; and as an Oral communication (\#CO-16) at the XIV SEOM National Congress held from 23 to 25 October 2013 in Salamanca, Spain

\section{Declaration of interest}

This study was supported by Sanofi. A Del Campo (Pivotal SL) provided medical writing services on behalf of Sanofi Pharmaceuticals. P Maroto has taken part in advisory boards for Sanofi, Jansen, Astellas, and Amgen. E Esteban has taken part in advisory boards for Sanofi, Pfizer, Novartis, and Roche and all retributions are given to FUNDESO (Foundation for Research and treatment of cancer). The other authors declare that they have no competing interests. ClinicalTrials.gov identifier: NCT01254279. 


\section{Bibliography}

Papers of special note have been highlighted as either of interest $(\bullet)$ or of considerable interest $(\bullet \bullet)$ to readers.

1. Vrignaud $\mathrm{P}$, Semiond D, Lejeune $\mathrm{P}$, et al. Preclinical antitumor activity of cabazitaxel, a semisynthetic taxane active in taxane-resistant tumors. Clin Cancer Res 2013;19:2973-83

2. Darshan MS, Loftus MS, Thadani-Mulero M, et al. Taxane-induced blockade to nuclear accumulation of the androgen receptor predicts clinical responses in metastatic prostate cancer. Cancer Res 2011;71:6019-29

3. Zhu ML, Horbinski CM, Garzotto M, et al. Tubulin-targeting chemotherapy impairs androgen receptor activity in prostate cancer. Cancer Res 2010;70:7992-8002

4. de Bono JS, Oudard S, Ozguroglu M, et al. Prednisone plus cabazitaxel or mitoxantrone for metastatic castration-resistant prostate cancer progressing after docetaxel treatment: a randomised open-label trial. Lancet 2010;376:1147-54

-• Pivotal study that demonstrates the efficacy of cabazitaxel in metastatic castrate-resistant prostate cancer.

5. Smith TJ, Khatcheressian J, Lyman GH, et al. 2006 update of recommendations for the use of white blood cell growth factors: an evidence-based clinical practice guideline. J Clin Oncol 2006;24:3187-205

-• An international consensus that establishes the criteria for the administration of G-CSF in clinical practice.

6. Aapro MS, Bohlius J, Cameron DA, et al. 2010 update of EORTC guidelines for the use of granulocyte-colony stimulating factor to reduce the incidence of chemotherapy-induced febrile neutropenia in adult patients with lymphoproliferative disorders and solid tumours. Eur J Cancer $2011 ; 47: 8-32$

- A European consensus that establishes the criteria for the administration of G-CSF in clinical practice.

7. National Health Institute. National Cancer Institute Common Terminology Criteria for Adverse Events v4.0. NIH publication; 29 A.D. Report No.: \#09-7473. Available from: http://ctep.cancer.gov/protocolDevelopment/electronic_applications/ctc.htm\#ctc_30 [Last accessed: 20 June 2014]

8. Brown E. Medical Dictionary for Regulatory Activities (MedDRA®), in pharmacovigilance. In: Mann RD, Andrews EB, editors. Pharmacovigilance. 2nd edition. John Wiley \& Sons, Ltd, Chichester, UK; 2007. Available from: http://www.meddra.org [Last accessed: 20 June 2014]

9. De Velasco G, Anton Aparicio L, Esteban E. Cabazitaxel in patients with advanced CRPC after docetaxel failure: results of expanded program access (EAP) in Spain: safety and efficacy. J Clin Oncol 2012;30:abstract \#e15149

10. National Comprehensive Cancer Network. NCCN Clinical Practice Guidelines in Oncology: cancer-related fatigue. In: National Comprehensive Cancer Network, editor. Version 1.2013. ed Fort Washington, Pa: 2013. http://www.nccn.org/professionals/physician_gls/f_guidelines.asp\#fatigue. Accessed May 2013

11. Di Lorenzo G, D'Aniello C, Buonerba C, et al. Peg-filgrastim and cabazitaxel in prostate cancer patients. Anticancer Drugs 2013;24:84-9

12. Sperlich C, Saad F. Optimal management of patients receiving cabazitaxel-based chemotherapy. Can Urol Assoc J 2013;7:S18-24

13. Bahl A, Masson S, Malik Z. Cabazitaxel for metastatic castration-resistant prostate cancer (mCRPC): interim safety and quality-of-life (QOL) data from the U.K. early access program (NCT01254279). J Clin Oncol 2012;30:abstract \#44

14. Bracarda S, Gernone A, Gasparro D, et al. Real-world cabazitaxel safety: the Italian early-access program in metastatic castration-resistant prostate cancer. Future Oncol 2014;10(6):975-83 
- Presents similar experiences with Cabazitaxel within the same expanded access program (EAP)/compassionate use program (CUP) program in Italy.

15. Heck MM, Hoppner M, Horn T, et al. [Compassionate use of abiraterone and cabazitaxel: first experiences in docetaxel-pretreated castration-resistant prostate cancer patients]. Urologe A 2012;51:390-7

16. Heidenreich A, Scholz HJ, Rogenhofer S, et al. Cabazitaxel plus prednisone for metastatic castration-resistant prostate cancer progressing after docetaxel: results from the German compassionate-use programme. Eur Urol 2013;63:977-82

- Presents similar experiences with Cabazitaxel within the same EAP/CUP program in Germany.

17. Sridhar SS, Winquist E, Hubay S. Cabazitaxel early access program (EAP) - Canadian interim results: safety, QOL, and utility values in metastatic castration resistant prostate cancer (mCRPC). Ann Oncol 2012;23:abstract \#960

18. Wissing MD, van Oort IM, Gerritsen WR, et al. Cabazitaxel in patients with metastatic castrationresistant prostate cancer: results of a compassionate use program in the Netherlands. Clin Genitourin Cancer 2013;11:238-50

19. Heidenreich A, Bracarda S, Mason M, et al. Safety of cabazitaxel in senior adults with metastatic castration-resistant prostate cancer: results of the European compassionate-use programme. Eur J Cancer 2014;50:1090-9

- Presents the preliminary results of the whole EAP/CUP program, with special focus in the elderly population.

20. Armstrong AJ, Garrett-Mayer ES, Yang YC, et al. A contemporary prognostic nomogram for men with hormone-refractory metastatic prostate cancer: a TAX327 study analysis. Clin Cancer Res 2007; 13:6396-403

21. Halabi S, Small EJ, Kantoff PW, et al. Prognostic model for predicting survival in men with hormone-refractory metastatic prostate cancer. J Clin Oncol 2003;21:1232-7

22. Bahl A, Oudard S, Tombal B, et al. Impact of cabazitaxel on 2-year survival and palliation of tumour-related pain in men with metastatic castration-resistant prostate cancer treated in the TROPIC trial. Ann Oncol 2013;24:2402-8

- Provides an interesting subanalysis in the elderly from the pivotal study TROPIC. 